\title{
High-Throughput SEM via Multi-Beam SEM: Applications in Materials Science
}

\author{
Joseph R. Michael ${ }^{1}$, Craig Y. Nakakura ${ }^{1}$, Tomasz Garbowski ${ }^{2}$, Anna Lena Eberle ${ }^{2}$, Thomas Kemen ${ }^{2}$ and \\ Dirk Zeidler ${ }^{2}$ \\ 1. Sandia National Laboratories, Albuquerque, NM 87185 \\ 2. Carl Zeiss Microscopy GmbH, Oberkochen, Germany
}

The maximum image acquisition rate for conventional SEM is limited by many factors related to both the instrumentation used and the physics of secondary electron image formation. The instrumentation limitations are related to maximum scan rates, detection band width and collection efficiency. The signal-to-noise for a given pixel dwell time is dependent mainly upon the beam current of the primary beam. For a given beam current, shorter pixel dwell time results in a reduction in the signal-to-noise ratio for the image. Thus, the only way to have higher throughput is to continually increase the beam current or improve the collection efficiency of the detectors employed. However, it is not possible to increase the beam current continuously due to the eventual loss of resolution associated with electronelectron interactions contributing to beam broadening and to electron optical considerations that require larger convergence angles and further increase in the beam size. Even if the desired resolution could be achieved with higher beam currents, there are some classes of samples that will be damaged or modified by high current imaging. This paper will introduce the multi-beam SEM and then discuss recent advances in its applications for high throughput imaging of materials science samples.

Improvements in imaging rate in the SEM can be achieved by use of multiple-beam SEM where an array of electron beams is scanned across a sample to achieve large area imaging while maintaining the required resolution.[1-3] A schematic of the multiple-beam SEM optics is shown in Figure 1. The multiple-beam SEM produces an array of 61 electron beams from a micro-optic element that is illuminated by a single Schottky electron source. The 61 beams are focused on to the sample surface. Secondary electrons generated by the individual beams are collected with very high efficiency and focused into individual beams of electrons that are normal to the sample surface. The secondary electrons generated by each beam are projected through a second electron column onto a multi-detector array and the signal from all the beams is collected simultaneously. Careful design and alignment of the projector column has reduced cross talk between the imaging detectors to levels that are acceptable. A magnetic beam splitter is utilized to separate the primary and secondary electron beams. The total data throughput rate is then the product of the single detector collection rate with the number of electron beams used. In the present case, an array of 61 beams scanned over the sample surface produces images at up to 1.2 gigapixels per second.

Practical imaging is achieved by simultaneously scanning all 61 beams such that each beam scans a subset of the total image area while including a small overlap between the images. Thus, large areas are scanned in the time it takes to scan one sub-image leading to the high throughput. Imaging of larger areas is achieved by stepping the sample stage across the area to be imaged, acquiring an image at each step, then stitching the images together. In this manner, large areas $\left(\mathrm{mm}^{2}\right)$ can be rapidly imaged at a pixel size as small as $3 \mathrm{~nm}$. Figure 2 is an image of a $\mathrm{Au}$ on $\mathrm{C}$ resolution sample obtained at $3 \mathrm{kV}$ with a single electron beam and demonstrates a resolution of $2 \mathrm{~nm}$. Figure 3 is a multi-field of view (mFOV) of a static random access memory (SRAM) test structure.[4] This image is about $100 \mu \mathrm{m}$ in width and contains 61 separate images all obtained in parallel in 1 second with a pixel size of $4 \mathrm{~nm}$. Figure 4 
demonstrates the detail visible in the individual images of the test structure. Potential important applications of high throughput imaging are in the areas of quality control, failure analysis and the potential to study evolving structures over large areas with good temporal resolution.

\section{References}

[1] A. L. Keller et al, Proc. of SPIE, 9236 (2014), 92360B-1.

[2] A. L. Eberle et al, Microscopy (Tokyo), 63 (2104),1.

[3] A. L. Eberle et al, J. Microsc. Accepted for publication, 2015.

[4] http://www.brukerafmprobes.com/a-3553-scmsample.aspx.

[5] Sandia is a multiprogram laboratory operated by Sandia Corporation, a Lockheed Martin

Company, for the United States Department of Energy (DOE) under contract DE-AC0494AL85000.



Figure 1. Schematic of the $\mathrm{mSEM}$ electron optics. Figure 2. Au on $\mathrm{C}$ image acquired at $3 \mathrm{kV}$.

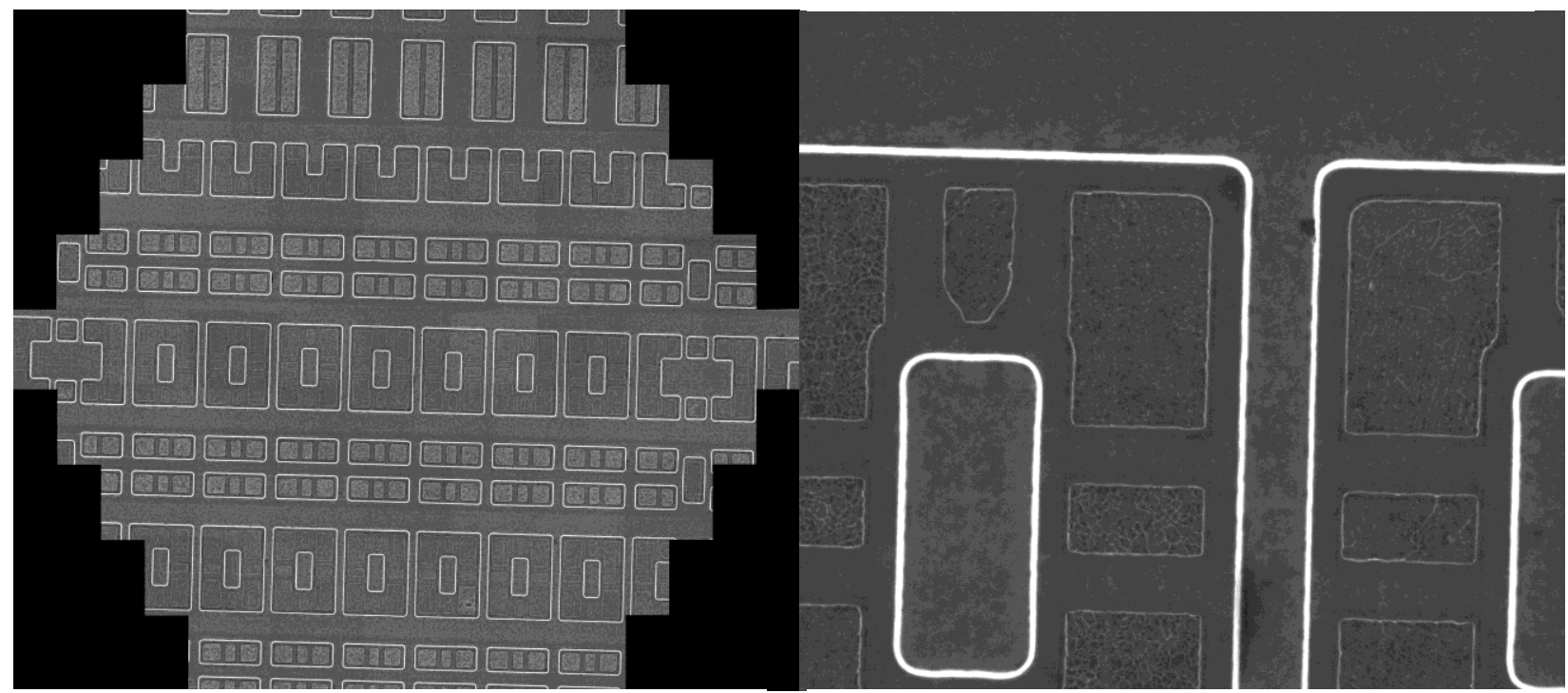

Figure 3. mFOV of an SRAM test structure acquired at $1.5 \mathrm{kV}$ with $4 \mathrm{~nm} /$ pixel.
Figure 4 Imaged formed by the central beam from the mFOV in Figure 3. The width of the image is $12.6 \mu \mathrm{m}$. 